\title{
The Path to Wearable Ultrafiltration and Dialysis Devices
}

\author{
Edward F. Leonard ${ }^{a}$ Stanley Cortell ${ }^{b}$ James Jones $^{b}$

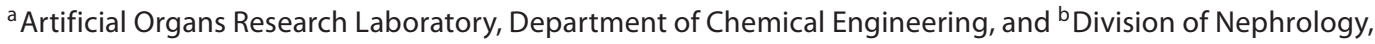 \\ St. Luke's-Roosevelt Hospital Center, Department of Medicine, Columbia University, New York, N.Y., USA
}

\section{Key Words}

Wearable devices • Ultrafiltration • Dialysis ·

Anticoagulation $\cdot$ Blood access

\begin{abstract}
Wearable blood processing devices offer an attractive solution to problems inherent in clinic-based, intermittent endstage renal disease therapies. What is involved in transitioning even a part of the current clinic-based population to ambulatory therapy has not been clearly enumerated. This paper addresses what a first-generation wearable device might accomplish, how issues of safety will need to be addressed, and what will make the device attractive to, and manageable by, the patient. Medical, technological, and economic issues are identified.
\end{abstract}

Copyright $\odot 2011$ S. Karger AG, Basel

The double-edged sword of clinic-based end-stage renal disease (ESRD) therapy has hung above the head of society for nearly 50 years. Millions of years of life extension have been delivered. However, current costs stagger many national health-care budgets. Current therapy imposes a large burden on all patients and an insupportable burden on some. Connecting to but reaching beyond these burdens, is a questionable quality of life within the years of extended life. Becoming free of the clinic is at- tractive to patients for many reasons including: (1) the physiological discomfort and disquietude of intense intermittent therapy that cycles without end; (2) the regimentation of a fixed often inconvenient schedule, communal transportation, and an imposed, nonprivate treatment environment not always with a preferred therapist; (3) the sheer loss of time is for many patients enhanced by the cyclicity of a treatment that devalues time before and after treatment and severely inhibits rehabilitation. These problems are widely recognized. They have been addressed with a variety of stationary therapeutic systems and by ambulatory peritoneal dialysis.

That wearable devices offer a compelling advantage over such solutions is completely unclear. However, all solutions to the limitations of in-clinic therapy present problems of cost, burdens of self-care and involvement of nonprofessional caregivers, and, at the root of it all, surveillance and management of a complex therapy and a complex disease. This paper simply deals with the possible role of wearable devices in this panoply of ways to mitigate current therapy.

The revolutionary nature of a clinically significant ambulatory blood processor is not to be underestimated. Given the short time between necessary replacements of at least some components in any existing, clinically significant blood processing device, it is inconceivable that any ambulatory device would first be used intracorporeally. On the other hand, extracorporeal circulatory assist

\section{KARGER}

두 2011 S. Karger AG, Basel

Fax +41613061234

E-Mail karger@karger.ch

www.karger.com
Accessible online at:

www.karger.com/bpu
Edward F. Leonard

Artificial Organs Research Laboratory

Columbia University, 500 West 120 St.

New York, NY 10027 (USA)

Tel. +1 212854 4448, Fax +1 212854 3053, E-Mail leonard@ columbia.edu 
devices have been developed and used successfully with ambulatory patients [1].

An extracorporeal ambulatory device needs, as is the case with any intervention, to be efficacious, safe and acceptable to the patient.

Efficacy is not so simple, and distinct proposals have been made at three levels. (1) Those that go beyond any therapeutic goal now addressed and seek to emulate both the ultrafiltration and resorption characteristics of the natural kidney, producing a urine-like output [2]. (2) Hemodialysis and ambulatory peritoneal dialysis systems that depend either upon sorbents, intermittent stationbased dialysate, or an ambulatory dialysate supply, i.e. a full replacement of in-clinic dialysis [3]. (And it must be remembered that CAPD eponymously, and in fact, is such a therapy [4].) (3) A system that aims only to maintain constant euvolemia and reduce the frequency of inclinic solute removal [5].

Safety is a difficult topic, in part because of the long tradition of rendering ESRD therapy under clinical supervision, and, even when that is suspended as in home dialysis, maintaining a controlled therapeutic environment. An ambulatory patient is at risk of exsanguination, hypotensive incidents, device-caused thromboembolic events, infection, and inappropriate alteration of blood composition.

Patient acceptability cannot be applied uniformly to the present dialysis population. Many of the problems associated with in-clinic therapy are ameliorated by home dialysis. Home dialysis is more like in-clinic treatment than is ambulatory therapy, and it may be perceived as safer [6]. A wearable device will often be worn all the time, which in a fully rehabilitated patient may be incompatible with certain activities such as swimming. On the other hand, non-ambulatory therapy will normally require connecting and disconnecting a device to the patient's circulation, which can be a demanding and unpleasant task. In-home dialysis may intrude noticeably on available living space although recent innovations reduce equipment size. Equipment associated with a patient receiving a mbulatory therapy can be maintained at a 'walkin' site while maintenance of home-based equipment requires a service call. The balance between ambulatory therapy and alternate treatment modalities will clearly be influenced by the size and unobtrusiveness of the ambulatory device. Overall, ambulatory therapy will place more control, and more responsibility, on the patient. It is almost certain that initial choices of ambulatory therapy will be made by patients who aggressively seek greater rehabilitation and are more risk tolerant.

Wearable Ultrafiltration and Dialysis Devices
In the remainder of this paper, the common technical problems needed to achieve an efficacious, safe and acceptable ambulatory therapeutic intervention will be defined and discussed.

\section{Minimum Extracorporeal Flow}

Adequate solute clearance is commonly defined by a prescribed Kt/V for urea. If a minimum value of 3.6 per week is taken, if the patient's urea pool is, say, 45 liters, and if therapy is provided in an absolutely continuous fashion, a clearance of about $16 \mathrm{ml} / \mathrm{min}$ is required. To allow for incomplete solute extraction and less than fulltime effective operation, a minimum blood flow of perhaps $30 \mathrm{ml} / \mathrm{min}$ is required. A volume extraction of $1 \mathrm{ml} /$ min is equivalent to $10 \mathrm{~kg} /$ week, a reasonable minimum requirement. For a device required only to maintain euvolemia, an extracorporeal blood flow might be as low as $10-15 \mathrm{ml} / \mathrm{min}$.

\section{Dialysate Requirement}

A daunting requirement placed on wearable devices is the need for a medium to receive solutes extracted from the blood flow. If this medium is to be dialysate, it will need to exceed the volume of blood flowed during the week, i.e. to be in excess of 300 liters. Substitution of sorbent cartridges for dialysate has been proposed, but it seems that their volume and cost would be very high.

\section{Blood Access}

Continuous extracorporeal blood flows have been maintained in small patient groups for many years. The Quinton-Dillard-Scribner shunt (fig. 1) developed at the University of Washington in the 1960s created a short extracorporeal flow loop that was intermittently accessed for dialysis $[7,8]$. One patient utilized this shunt for 35 years with only one declotting episode. Success with internal shunts led to our current use of arteriovenous fistulas and grafts [9]. Recent improvements in safety needles have allowed for safe overnight access. Central venous catheter systems have been used intermittently with some success [10]. Many total parenteral nutrition systems infuse solutions into the circulation continuously over long periods of time through subcutaneous ports [11].

A critical issue associated with blood access is thromboembolism. Virchow's triad (flow, surface, blood condition) applies once again. To maintain adequate flow velocities at the low flow rates indicated above, small cannulae will be required, and this will increase the surface to volume ratio in cannulae and devices. Continuing de- 
velopment of passive or even actively antithrombotic surfaces will continue to be required. Blood condition may be modified by anticoagulation. However, continuous, systemic, whole-body anticoagulation of ambulatory patients seems likely to lead to a greatly increased incidence of accidental and occult bleeding. Heparin infused into a low-flow extracorporeal circuit can achieve higher concentrations than those expected systemically. If one assumes heparin clearance to be invariant with heparin concentration, and blood returning from the extracorporeal circuit to be quickly mixed, the ratio of extracorporeal concentration $c_{E}$ to systemic concentration $c_{S}$ is equal to the ratio of extracorporeal flow $\mathrm{Q}_{\mathrm{E}}$ to hepatic clearance, $\mathrm{K}_{\mathrm{H}}$. Thus, for a heparin clearance of $100 \mathrm{ml} / \mathrm{min}$ and an extracorporeal flow of $30 \mathrm{ml} / \mathrm{min}$, the systemic heparin concentration might be about three times lower. It is not clear, however, that this difference affords an adequate margin of safety.

\section{Component Performance}

All blood purification devices, fixed or ambulatory, depend upon the selective movement of blood components across an artificial blood-contacting surface. Such surfaces activate blood components and are themselves transformed by blood contact. If no transport occurs through the surface, it is sufficient that the surface achieves a stable interface that does not occlude the blood flow path, does not embolize, and does not deteriorate. When, as in all practical therapeutic devices, transport occurs through the surface the requirements imposed upon the interface increase. First, material that will not penetrate the surface accumulates there, creating a layer that impedes transport. Second, over time material may invade the transport paths through the surface, reducing the rate at which transport occurs - and not to a lesser degree in exotic new materials [12]. These surface-fouling phenomena [13] are noticeable during short and extended treatments but are likely to increase steadily over time. No transport surface that remains nonfouling over extended times has yet been demonstrated. Some method of 'swapping out' transport surfaces in ambulatory devices will thus be required. Data that indicate how quickly blood and plasma-wetted transport surfaces foul are lacking. These data are likely to depend sharply upon rates of flow over and through the transport surfaces.

\section{Device Size and Placement}

The relationship among blood flow, dialysate flow, and transport area in ambulatory devices follow longstanding analytical formulae, but the low blood flow and

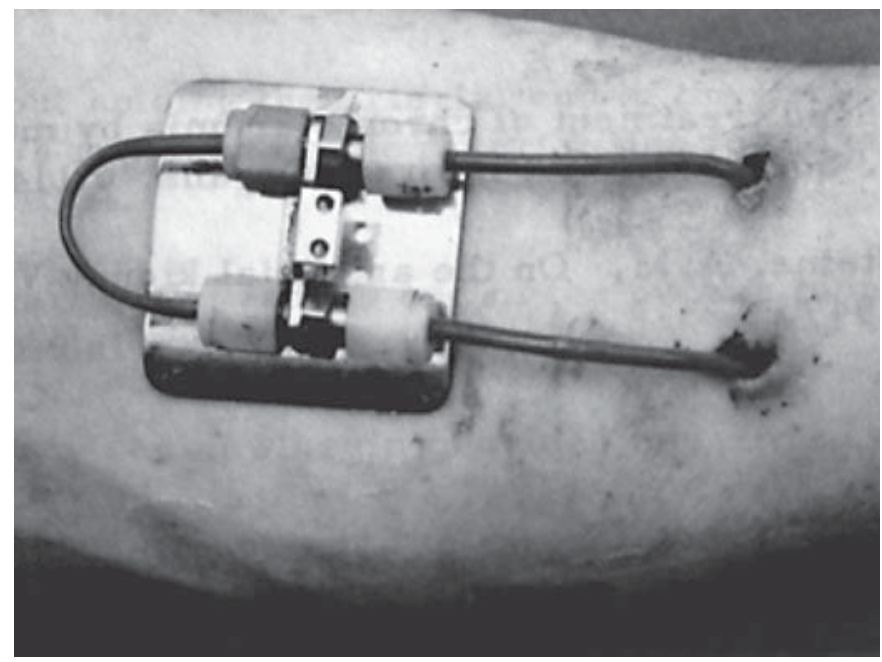

Fig. 1. Quinton-Dillard-Scribner exteriorized Teflon arteriovenous shunt. This type of shunt was worn by patient James Albers for 35 years with one declotting event during that period. Christopher R. Blagg, personal communication.

special requirements associated with ambulatory therapy make for different design choices. Dialysate flow can be lowered nearly to that of blood if transport area is increased enough. Or, if desired, the size of the physical device can be lessened if compensation for smaller transport areas is obtained with somewhat larger blood flows. Concerns about blood access auger for venovenous blood access systems, and this choice mandates the use of a blood pump. Only a few types of small lightweight pumps are available, and most are pulsatile. Peristaltic pumps that keep blood within a tube and do not require a separate pump chamber are desirable because they keep the pump mechanism and the fluid stream on opposite sides of a tube. Gura et al. [14] describe a double-headed pump that moves both blood and dialysate and uses its pulsatility to increase transport rates in a dialyzer. However, small peristaltic pumps typically exhibit a higher normalized index of hemolysis (NIH). NIH values much above $15 \mathrm{~g}$ hemoglobin released per 100 liters of blood pumped are probably unacceptable.

A pumped blood flow requires power. While the theoretical power required to pump $30 \mathrm{ml} / \mathrm{min}$ through a pressure difference of, say, 50 torr is negligible (less than $0.01 \mathrm{~W}$ ), peristaltic pump efficiencies are low. Modern lithium-ion batteries have energy densities on the order of 0.1 watt-hours/gram. At reasonable pump efficiencies, batteries weighing less than $100 \mathrm{~g}$ should be able to circulate blood for a full $24 \mathrm{~h}$ without recharge. 
The question of device size is closely connected to utility and the patient's passion for ambulatory therapy. A device attached to a motorized wheelchair could be massive but might still be attractive to a small subset of the patient population. An unnoticeable stand-alone device attached to the body would need to weigh less than $1 \mathrm{~kg}$ and have a volume less than $1,000 \mathrm{~cm}^{3}$. The question of device density and shape should be resolved so that the device is comfortable and moves with the body even under the high accelerations encountered in accidental circumstances. Placement is clearly related to the location of the blood supply.

\section{Safety}

A wearable device that exteriorizes blood and exposes it to permeable surfaces is essentially unprecedented. Stationary blood purification devices are partial precedents. Safety issues will involve regulatory agencies and regulatory clearance will be facilitated by identifying predicate devices along with their safety systems. Critical components will thus include bubble and leak detectors, pressure monitors, and some indication of deviation from intended flows. It is likely that alarming will be an insufficient response to unsafe conditions and that some form of automatic shutdown will be required.

There are imponderable safety issues that may be mitigated by design or controlled by monitoring additional variables. Long experience with ambulatory circulatory support devices points up the 'little things' that determine whether a device is safe or not.
How to Get There from Here?

While the history of scientific and engineering innovation touts revolutionary changes, most innovations are evolutionary. Some are completely successful and the final outcome is indeed revolutionary - but only eventually.

Fundamental changes will come slowly for many reasons: Treatment of the world's 2 million ESRD patients is highly organized, the result of an immense investment in material and professional capital. ESRD is a complex syndrome whose management cannot be thrust upon a patient simply because he is enabled to walk about with his therapy. Device development is still at the point where frequent maintenance, especially of transport surfaces, is necessary. Almost certainly ambulatory ESRD therapy will require that the patient have clinical contact every few days for medical surveillance and device maintenance. Even though increased rehabilitation is likely to reduce real costs eventually, progress toward it is likely to occur under severe cost limitations.

The clinic is certainly not a bad place, and a responsible liberation of patients from its strictures will probably take place in small steps, slowly.

This paper is dedicated to the memory of Prof. Michael Lysaght who, had he lived, might well have said it better.

\section{Acknowledgement}

The authors are grateful for the support of the National Institutes of Health (grant No. 1R21HL088162) and Vizio Medical Devices LLC.

\section{References}

1 Geidl L, Zrunek P, Deckert Z, Zimpfer D, Sandner S, Wieselthaler G, Schima H: Usability and safety of ventricular assist devices: human factors and design aspects. Artif Organs 2009;33:691-695.

-2 Fissell WH, Fleischman AJ, Humes HD, Roy S: Development of continuous implantable renal replacement: past and future. Transl Res 2007;150:327-336.

-3 Gura V, Beizai M, Ezon C, Polaschegg HD: Continuous renal replacement therapy for end-stage renal disease - the wearable artificial kidney (WAK); in Ronco C, Brendolan A, Levin NW (eds): Cardiovascular Disorders in Hemodialysis. Contrib Nephrol. Basel, Karger, 2005, vol 149, pp 325-333.

$\checkmark 4$ Winchester JF, Amerling R, Harbord N, Capponi V, Ronco C: The potential application of sorbents in peritoneal dialysis; in Ronco C, DellAquila R, Rodighiero MP (eds): Peritoneal Dialysis: A Clinical Update. Contrib Nephrol. 2006, vol 150, pp 336-343.

5 Leonard EF: Technical approaches toward ambulatory ESRD therapy. Semin Dial 2009 22:658-660.

6 Sands JJ, Lacson E, Ofsthun NJ, Kay JC, Diaz-Buxo JA: Home hemodialysis: a comparison of in-center and home hemodialysis therapy in a cohort of successful home hemodialysis patients. Asaio J 2009;55:361368 .

7 Gallieni M, Martini A, Mezzina N: Dialysis access: an increasingly important clinical issue. Int J Artif Organs 2009;32:851-856.

8 Chan MR, Yevzlin AS: Tunneled dialysis catheters: recent trends and future directions. Adv Chronic Kidney Dis 2009;16:386-395.

$\checkmark 9$ Sansivero GE: Features and selection of vascular access devices. Semin Oncol Nurs 2010;26:88-101.
10 Berardinelli L: The endless history of vascular access: a surgeon's perspective. J Vasc Access 2006;7:103-111.

11 Konner K: Vascular access in the 21st century. J Nephrol 2002;15(suppl 6):S28-S32.

12 Al-Amoudi AS: Factors affecting natural organic matter (NOM) and scaling fouling in NF membranes: a review. Desalination 2010; 259:1-10.

13 Kanani DM, Fissell WH, Roy S, Dubnisheva A, Fleischman A, Zydney AL: Permeabilityselectivity analysis for ultrafiltration: effect of pore geometry. J Memb Sci 2010;349:405410.

14 Gura V, Beizai M, Ezon C: Convective forces across the membrane with opposite phase double pulsation flow in the wearable artificial kidney (WAK). Blood Purif 2008;26: 440-440. 